\title{
Acute food refusal in a seven year old hospitalised boy
}

\begin{abstract}
Avoidant/Restrictive Food Intake Disorder (ARFID) is a new category of eating disorders in DSM-5. Management of children with ARFID varies between clinicians with no available empirical data to support the efficacy or superiority of any intervention. The case reported here describes the management of a hospitalised seven-year-old boy with ARFID considering the influence of stress related to hospital admission on the course of illness.
\end{abstract}

Keywords: avoidant restrictive food intake disorder, eating disorder, non organic food refusa
Volume 8 Issue 6 - 2018

\author{
Dalia Albahari, Mohamed Rabie \\ Alwakra Hospital, Hamad Medical Corporation, Qatar
}

Correspondence: Dalia Albahari, Psychiatry specialist in consultation and liaison psychiatry, Alwakra Hospital, Hamad Medical Corporation, Qatar, Email daliabahary@hotmail.com

Received: October 26, 2017 | Published: November 06, 2018

\section{Case report}

A seven year old boy of an East-African descend was brought by his parents to the Pediatric Emergency Centre (PEC) with diffuse abdominal pain. His mother reported fever, cough, abdominal pain, and constipation in the past couple of days. The patient initially responded well to paracetamol, but the abdominal pain persisted. The mother was concerned about reduced oral intake. The patient's physical examination was unremarkable. His weighed between 15 th 50th percentiles. An abdominal X-ray showed fecal material and gas. The patient was treated with an enema and was discharged home on laxatives. He was brought back to the PEC 18 hours later; parents were concerned that the patient completely refused oral intake, continued to complain of abdominal pain and appeared tired and a pathetic. The patient did not experience fever, vomiting, diarrhea or any previous incident of choking or swallowing difficulties. Clinical examination showed signs of dehydration but otherwise was unremarkable. Further blood testing revealed the following results: (random blood glucose $1.5 \mathrm{mmol} / \mathrm{l}, \mathrm{pCO}_{2} 12.5 \mathrm{mmHg}$ and $\mathrm{pH} 7.22$, Lactic acid $1.2 \mathrm{mmol} / \mathrm{L}$, Betahydroxybuterate: $5.8 \mathrm{mmol} / \mathrm{L}$, Insulin $3.4 \mathrm{mc}$ unit $/ \mathrm{mL}$, Human Growth Hormone $(\mathrm{HGH})=4.95 \mathrm{mcg} / \mathrm{L}$, Cortisol pm $362 \mathrm{nmol} / \mathrm{L}$, Bicarbonate $=10 \mathrm{mmol} / \mathrm{L}$ and Chloride: $114 \mathrm{mmol} / \mathrm{L}$ ). The working diagnosis was ketotic hypoglycemia secondary to starvation, and the patient was treated with intravenous infusion of $10 \%$ dextrose. The patient's blood glucose dropped every time the intravenous dextrose infusion was stopped. The patient was admitted to the hospital for stabilisation. Pain management using paracetamol and scopolamine were not effective. He was kept on full maintenance of intravenous fluids as efforts to encourage oral intake were not successful The patient's blood tests had later normalized. The abdominal ultrasonography exam was normal. The food allergy and celiac screen tests were negative.

The parents and the staff tried positive reinforcement and punishments to encourage oral intake, but this appeared to have high tended the levels of emotions during meal time and did not motivate the patient to eat or drink. He became more withdrawn and less engaged with staff, and he requested that no food to be brought into the room as he could not tolerate its smell.

To reduce the risk of malnutrition, a nasogastric tube feeding $(\mathrm{NG})$ was inserted for intermittent feeding of the daily caloric requirement. This made the patient more distressed, and he was reported to cry during the feeding sessions and complaint of abdominal pain. Upper GI-endoscopy was performed under general anesthesia, and no abnormalities were detected.

\section{Psychiatry consultation}

On the fourth day of admission, the pediatric team requested input from the hospital consultation- liaison psychiatry team for evaluation of non-organic causes of food refusal. Despite that the parents and the child felt uncomfortable initially about the psychiatry team involvement, they eventually agreed after a prolonged discussion with the pediatric team. Such reluctance may reflect the stigma that surrounds mental health in the region. Parents also felt that the proposal of the psychiatric assessment should not have been discussed in the presence of the child.

On the initial psychiatry assessment, the patient was lying on the bed watching cartoons, he was observed to show fair interest in the show but not to the team. It took some time for him to warm up and engage in the conversation. His speech was low in volume with a monotonous tone, eye contact was poor initially but improved towards the end of the interview. The patient denied feeling sad or anxious, he reported that he was admitted to the hospital before, but he does not understand why he is in the hospital this time. He refused offers of food by the interviewer without giving a reason. On direct questioning, he reported epigastric pain which was associated with eating in the past and is associated with the NG feeding currently. He did not want the NG tube and requested that the team removes it. The patient did not display any distorted self-image thoughts or a desire to lose weight. The patient expressed his wish to be discharged from the hospital many times during the interview; he mentioned that he missed his siblings and wanted to play with them. There were no abnormal thoughts elicited during the assessment. The patient was revisited by the team the day after the gastroscopy procedure; he was observed to become more distressed during feeding times, he was mute during interaction with staff and was only verbal when alone with his mother.

\section{Personal and medical history}

The patient's parents had settled in Qatar 17years ago; he is the youngest of four children. The pregnancy was uneventful, and the patient's mile stones were normal. The patient did not experience any 
traumatic events or abuse in the past. The patient suffers asthma with frequent presentations to the emergency department. He presented to PEC with respiratory tract infections and asthma attacks five times in the four weeks before his current admission. He was not on regular medications apart from salbutamol inhaler for the acute attacks. His academic achievement was reasonable despite missing lots of school days due to frequent illnesses. He had just started in a new school and had difficulties adjusting while having to miss three weeks in the first three months of school. His mother described him as a shy child that can become slightly anxious. His appetite was typically small, he tends to lose appetite and refuse food during acute illness but regain sit quickly when he starts to improve. He had never displayed any restrictive eating behavior before. The home environment appeared stable with no heightened emotions around food despite the parents concern about the patient's limited appetite compared to his siblings.

\section{Diagnosis and further management}

The patient meets criteria for Avoidant/Restrictive Food Intake Disorder (ARFID). ARFID diagnosis is a relatively new DSM-5 category of eating disorders; it occurs in patients exhibiting restrictive or avoidant eating behaviors that result in significant weight loss, growth compromise, a reliance on nutritional supplements to meet daily energy requirements, nutritional deficiency or marked interference with the patient's psychosocial functioning. ${ }^{1}$ ARDIF cannot be diagnosed in the presence of other mental illness, medical illness or cultural believes that could explain food refusal. Initial food refusal, in this case, can be traced to the aversive experience of abdominal pain, which was likely due to a viral illness. The patient associated and conditioned the pain with food intake. The stress associated with hospital admission and medical procedures has perpetuated and magnified the condition.

Management of this patient did not involve complex psychological interventions or psychotropic medications. The central component of the management aimed at decreasing such stress before addressing the process of feeding. Psycho education of parents and staff decreased their anxiety, reduced heightened emotions and provided a neutral environment for the patient. Despite parents' concerns about psychiatry involvement in the patient's management, the team managed to establish rapport with the patient and his parents which impacted his management positively. Positive reinforcement and punishment discontinued as these approaches were not successful with the patient and contributed to a tense environment.

The second step to decrease stress was by validating the psychological component of the patient's condition, this led to the minimisation of medical procedures, and no new investigations were pursued. Providing the patient with a familiar environment was proposed to decrease his stress. His environment was manipulated by giving the patient planned and gradual breaks from the hospital environment. The patient spent this time with family at home. As the patient developed hypoglycemia rapidly in the past when intravenous fluids stopped, there was great concern from the pediatric team about the patient's readiness to leave the hospital. To facilitate outings, the parents received education on the NG tube feedings and were asked to contact the emergency services if they noticed any signs or symptoms of hypoglycemia. The education of the parents helped to build up their confidence, and the first short outing of the patient was successful. The breaks from the hospital started to be regular and longer with the patient, the family and the medical team's confidence growing stronger.
The outings facilitated appetite manipulation and hunger provocation by temporarily interrupting NG feeding while outside the hospital as the patient started to refuse NG feeding at home and the family introduced small portions of solid food which he consumed. The NG feeding was reduced to stimulate patient's appetite and was eventually stopped once normal oral intake was ensured. With the improvement in appetite and the cessation of medical investigations, the patient was observed to be more cheerful, he engaged better with staff and observed to be enjoying his video games.

The patient was reviewed in the child and adolescent psychiatry outpatient clinic after discharge from hospital for approximately two months where he received play therapy and family interventions. He also attended the pediatric clinic for a follow up appointment two months later; food intake continued to be normal, and the patient gained $1.2 \mathrm{~kg}$ since discharge from the hospital.

\section{Discussion}

Despite that the prevalence of feeding problems in childhood is estimated to be around $25 \%$ of the population ${ }^{2,3}$ there is not a universally accepted terminology or definition for food refusal. Multiple diagnostic criteria for non-organic food refusal are used in the literature; terminology varies from food specific phobia, posttraumatic feeding problem, phagophobia of traumatic type to food avoidance emotional disorder. ${ }^{2,4-7}$ The DSM-5 has introduced a new eating disorder category (ARFID) which helped clinicians diagnose many children who did not fit into other categories of eating disorders. Unfortunately, there is no empirical evidence to provide clinical guidelines on the management of ARFID yet. Published cases of ARFID have described varieties of psychological interventions and pharmacological therapy which were tailored to the specific circumstances of each case. Psychological interventions have focused on the process of feeding and the dynamics of meal time as ARFID cases have the most difficulties with the feeding process rather than the outcome which is weight gain.

Guvenek-Cokol and his colleagues have successfully treated an inpatient five year old girl with ARFID. ${ }^{8}$ The food refusal, in this case, was triggered by an aversive experience, and it was perpetuated by traumatic medical stress inflicted by prolonged hospital admission and medical procedures. A multidisciplinary team delivered the management plan with parents involved in the plan for consistency and sustainability of the results. In addition to optimising the child's environment to minimise stress and reduce medicalisation, a firm behavioral plan was employed aiming for a gradual increase of oral intake. The behavioural treatment focused on increasing appropriate feeding behavior along with decreasing maladaptive patterns. Attention to the importance of psychosocial component of the management of hospitalised children is recommended to prevent the acute stress of admitted children and their families. ${ }^{8}$ Pitt and Middleman have described two teenage patients with ARFID who were treated in the inpatient setting with a good outcome. The management focused on decreasing the focus on the medical aspect of the illness with enforcing an individualised behavioural plan for feeding behavior; the families received therapy as part of the management plan. ${ }^{9}$ Cognitive behavioural therapy was used to treat a 13year old child with ARFID by Bryant-Waugh, the treatment emphasised the role of the family for best outcomes. ${ }^{10}$

There is no available evidence to support pharmacological therapy in the treatment of ARFID yet. However, some clinicians have used a wide range of medications as part of integrated clinical management. 
Risperidone was used by Pennell and colleagues to enhance appetite and to increase weight in two complex cases of ARFID with comorbid Attention Deficit Hyperactivity Disorder on stimulants therapy. ${ }^{11}$ Mirtazepine has produced satisfactory outcomes in children with ARFID treated by Gray and his colleagues in comparison to other psychoactive medications. ${ }^{12}$

The literature describes various psychological interventions to treat non organic feeding problems that include, but not limited to, gradual exposure, escape prevention, relaxation techniques and cognitive reconstruction and self-management. Optimizing the environment and parental involvement are highly recommended for effective treatment. ${ }^{13,14}$ Many psychological theories are proposed to explain food refusal; learning theory provides one of the psychological explanation for food refusal and perhaps a foundation for management interventions too. Aversive and traumatic experiences that the child associates with food have a major role in perpetuating food refusal. Vomiting, incidents of choking and abdominal pain are some of the triggers for fear of feeding. ${ }^{15}$ Our patient was primarily managed by optimising the environment, mitigating the stress caused by hospitalisation and minimising medical interventions. Complex behavioural interventions were not used in the pediatric inpatient unit as neither the pediatric or the consultation liaison psychiatry teams have access to a child psychologist or eating disorder specialist staff. Lack of such staff may explain the failure of positive enforcement in producing the desired effect in this case despite consist reports in the literature of its efficacy. ${ }^{14}$ The acute nature of food refusal in our patient coupled with a clear association with the continuity of hospital admission are the factors that influenced the management plan of this patient. Patient has recovered in two weeks which could be considered a rapid recovery compared with other cases that had a longer duration of food refusal with chronic weight loss.

It is important to acknowledge the stress that hospitalisation could inflict on children and their families, the necessity of medical procedure has to be evaluated carefully, and any medical discussion needs to be delivered in a child sensitive way. Access to multidisciplinary team members to maintain children's psychological wellbeing during hospitalisation is essential for effective delivery of care. Role of early community based intervention in the management of ARFID needs further research as this may reduce hospitalisation time and stress associated with it.

Treating this patient was tailored around his specific circumstance but supported by the previous work published in similar cases, more research is needed to provide evidence based management approach for this group of children.

\section{Conflict of interest}

The author declares that there is no conflict of interest.

\section{References}

1. Norris M, Spettigue W, Katzman D. Update on eating disorders: current perspectives on avoidant/restrictive food intake disorder in children and youth. Neuropsychiatr Dis Treat. 2016;12:213-218.

2. Dovey TM, Farrow C V, Martin CI, et al. When Does Food Refusal Require Professional Intervention? Curr Nutr Food Sci. 2009;5(3):160 71 .

3. Nock MK. A multiple-baseline evaluation of the treatment of food phobia in a young boy. J Behav Ther Exp Psychiatry. 2002;33(3-4):217-25.

4. Baijens LWJ, Koetsenruijter K, Pilz W. Diagnosis and treatment of phagophobia: A review. Dysphagia. 2013;28(2):260-270.

5. Higgs, J F; Goodyer, I M; Birch J. Anorexia nervosa and food avoidance emotional disorder. Arch Dis Child. 1989;64(3):346-351.

6. Okada A, Tsukamoto C, Hosogi M, et al. A study of psycho-pathology and treatment of children with phagophobia. Acta Med Okayama. 2007;61(5):261-269.

7. Yang HR. How to approach feeding difficulties in young children. Korean J Pediatr. 2017;60(12):379-384.

8. Guvenek-Cokol PE, Gallagher K, Samsel C. Medical Traumatic Stress: A Multidisciplinary Approach for Iatrogenic Acute Food Refusal in the Inpatient Setting. Hosp Pediatr. 2016;6(11):693-698.

9. Pitt PD, Middleman AB. A Focus on Behavior Management of Avoidant/ Restrictive Food Intake Disorder (ARFID): A Case Series. Clin Pediatr (Phila). 2018;57(4):478-480.

10. Bryant-Waugh R. Avoidant restrictive food intake disorder: An illustrative case example. Int J Eat Disord. 2013;46(5):420-423.

11. Pennell A, Couturier J, Grant C, Johnson N. Severe avoidant/restrictive food intake disorder and coexisting stimulant treated attention deficit hyperactivity disorder. Int J Eat Disord. 2016;49(11):1036-1039.

12. Gray E, Chen T, Menzel J. Mirtazapine and Weight Gain in Avoidant and Restrictive Food Intake Disorder. J Am Acad Child Adolesc Psychiatry. 2018;57(4):288-289.

13. Herpertz-Dahlmann B. Treatment of eating disorders in child and adolescent psychiatry. Curr Opin Psychiatry. 2017;30(6):438-45.

14. Williams KE, Field DG, Seiverling L. Food refusal in children: A review of the literature. Res Dev Disabil. 2010;31(3):625-633.

15. Douglas J. Psychological Treatment of Food Refusal in Young Children. Child Adolesc Ment Health. 2002;7(4):173-180.

\section{Acknowledgements}

None. 